\title{
3D spectroscopy with VLT/GIRAFFE
}

\section{Angular momentum and dynamical support of intermediate redshift galaxies ${ }^{\star}$}

\author{
M. Puech ${ }^{1}$, F. Hammer ${ }^{1}$, M. D. Lehnert ${ }^{2}$, and H. Flores ${ }^{1}$ \\ ${ }^{1}$ Laboratoire Galaxies Étoiles Physique et Instrumentation, Observatoire de Paris, 5 place Jules Janssen, 92195 Meudon, France \\ e-mail: mathieu.puech@obspm.fr \\ ${ }^{2}$ Max-Planck-Institut für Extraterrestrische Physik, Giessenbachstraße, 85748 Garching, Germany
}

Received 5 July 2006 / Accepted 8 January 2007

\section{ABSTRACT}

\begin{abstract}
Context. One of the most outstanding problems related to numerical models of galaxy formation is the so-called "angular momentum catastrophe", i.e., the inability to theoretically explain the high angular momentum observed in local disk galaxies.

Aims. We study the evolution of the angular momentum from $z \sim 0.6$ to $z=0$ to better understand the mechanisms responsible for the large angular momenta of disk galaxies observed today. This study is based on a complete sample of $32,0.4 \leq z \leq 0.75$ galaxies observed with FLAMES/GIRAFFE at the VLT. Their kinematics had been classified as rotating disks (11 galaxies), perturbed rotators (7 galaxies), or complex kinematics (14 galaxies).

Methods. We have computed the specific angular momentum of disks $\left(j_{\text {disk }}\right)$ and the dynamical support of rotating disks through the $V / \sigma$ ratio. To study how angular momentum can be acquired dynamically, we compared the properties of distant and local galaxies, as a function of their kinematical class.

Results. We find that distant rotating disks have essentially the same properties ( $j_{\text {disk }}$ and $\left.R_{\mathrm{d}}\right)$ as local disks, while distant galaxies with more complex kinematics have a significantly higher scatter in the $j_{\text {disk }}-V_{\max }$ and $R_{\mathrm{d}}-V_{\max }$ planes. On average, distant galaxies show lower values of $V / \sigma$ than local galaxies, the lowest $V / \sigma$ values being reached by distant galaxies showing perturbed rotation. This can probably be attributed to heating mechanisms at work in distant disks.

Conclusions. We found observational evidence for a non-linear random-walk evolution of the angular momentum in galaxies during the past 8 Gyr. The evolution related to galaxies with complex kinematics can be attributed to mergers, but not to (smooth) gas accretion alone. If galaxies observed at intermediate redshift are related to present-day spirals, then our results match the "spiral rebuilding" scenario proposed by Hammer et al. (2005) quite closely.
\end{abstract}

Key words. galaxies: evolution - galaxies: formation - galaxies: kinematics and dynamics

\section{Introduction}

The evolution and the origin of the Tully-Fisher (T-F) relation is still a matter of intense debate. A strong evolution, both in slope and dispersion, has been found in the $B$ band (e.g., Ziegler et al. 2002; Böhm et al. 2004), from $z \sim 1$ to $z=0$. More recently, Conselice et al. (2005) derived the T-F relation in $K$ band, $K$-band absolute magnitude being a better tracer of stellar mass, and found such a large dispersion that one could wonder whether or not the T-F relation even exists at $z \sim 1$. Flores et al. (2006, hereafter Paper I) derived the first T-F relation for distant galaxies using integral field spectroscopy. The two-dimensional spatial coverage allowed them to properly identify the dynamical nature of distant galaxies and to establish that as much as $\sim 40 \%$ of field galaxies are not in equilibrium, i.e., galaxies that are not suitable to establishing a proper T-F relation. They then derived a T-F relation that does not appear to have evolved since $z \sim 0.6$, in the $M_{K}$ (or stellar mass) versus $V_{\max }$ plane.

On the other hand, the stellar mass density increases from $z=1$ to $z=0$ (e.g., Drory et al. 2005): assuming a rough $30 \%$ increase in stellar mass from $z=0.6$ to $z=0$, we should then see a $\sim 0.1$ dex shift along the $M_{*}$ axis between the local and

^ Based on FLAMES/GIRAFFE Paris Observatory Guaranteed Time Observations collected at the European Southern Observatory, Paranal, Chile, ESO No. 71.A-0322(A) and 72.A-0169(A). distant T-F relations. However, one should keep in mind that typical uncertainties usually associated with $M_{*}$ are also within $0.1-0.2$ dex (see Paper I). Another possibility is to assume some gas accretion from the intergalactic medium that would be directly converted into stellar mass: while $M_{*}$ increases, $V_{\max }$ could then increase at the same time, because the total mass of the system mainly depends on the rotational velocity. Then, both quantities could evolve in such a way that the resulting evolution in the T-F plane operates along the relation (e.g., Portinari $\&$ Sommer-Larsen 2006). More statistics and/or future studies of the T-F relation at higher redshifts (where the stellar mass density was much lower than at $z=0.6$ ) should provide a decisive test of this point. Whether the T-F relation evolves with time will no doubt provide important clues about the relationship between the growth of mass and the characteristics of the stellar populations, and, ultimately, about galaxy formation and evolution in general.

The ideas concerning the origin of the T-F relation can be divided into two broad categories. In the first one, the T-F relation originates from the cosmological equivalence between the halo mass and the circular velocity (e.g., Mo et al. 1998). The relation then comes from the fact that the finite age of the Universe imposes a maximal radius from where matter can be accreted to form a galaxy. The second broad categories of models invoked to explain the T-F relation is self-regulated star formation 
in disks of different masses (e.g., Silk 1997). However, numerical simulations taking both ingredients of gas accretion and selfregulation into account have not been able to reproduce all aspects of the T-F relation, such as the zero point (e.g., Steinmetz \& Navarro 1999). Many authors have suggested that feedback from massive star formation or active galactic nuclei could help to solve these discrepancies (see, e.g., Eisenstein \& Loeb 1996; Heavens \& Jimenez 1999; Steinmetz \& Navarro 1999).

Feedback has also been suggested for solving the so-called "angular momentum catastrophe" of the $\Lambda$-CDM model, i.e., the inability of simulations to reproduce disk galaxies with as much angular momentum than observed (e.g., Steinmetz \& Navarro 1999). Feedback has been proposed as a potential solution to this problem, which has been investigated in detail (e.g., Maller \& Dekel 2002; D’Onghia et al. 2006; Governato et al. 2006). Stateof-the-art numerical simulations, including the effects of AGN feedback driven by accreting gas onto a super-massive central black hole, show how a disk can re-form after the merging of two rotating gas-rich disks, with a sufficient and consistent amount of angular momentum (Robertson et al. 2005).

The mechanisms through which galaxies may have acquired their angular momentum has been discussed for many decades (e.g., Strömberg 1934; Hoyle 1951; Mestel 1963). The so-called "gravitational instability paradigm", independent of the details of the cosmology, predicts that the angular momentum of a protogalaxy should grow linearly with time due to tidal torques from interactions with neighboring structures, until it decouples from the Hubble flow (Peebles 1969; White 1984). In a more modern picture, galaxies form from infalling baryonic gas embedded in dark matter halos, and their angular momentum is then inherited from the halo (White \& Rees 1978; Fall \& Efstathiou 1980; Barnes \& Efstathiou 1987). After the end of the epoch during which tidal torquing is effective, subsequent evolution of the angular momentum takes place non-linearly, through a randomwalk process associated with mergers events and/or mass accretion (Vitvitska et al. 2002; Maller \& Dekel 2002; Peirani et al. 2004). This random-walk leads to a change in the angular momentum of the halos, with a more significant change (increase or decrease, depending on the geometry of the merger) during major mergers (Vitvitska et al. 2002; Peirani et al. 2004). For a given galaxy, it is in this way that major mergers are the main source of either positive or negative change in its angular momentum.

Relating the angular momenta of the halo and the disk is not at all straightforward. A reasonable assumption often made is that the specific angular momentum $j$ (i.e., the angular momentum per unit mass), is conserved during the collapse of the gas (Mestel 1963; Fall \& Efstathiou 1980). If we also assume that both gas and dark matter are mixed well in the proto-galaxy (Fall \& Efstathiou 1980), we get $j_{\text {disk }} \sim j_{\text {halo, }}$, and then models and simulations can reproduce several properties of local disk galaxies (e.g., Mo et al. 1998; Syer et al. 1999; van den Bosch 2001, 2002a; Tonini et al. 2006). However, some problems remain, such as the "mismatch of angular momentum profiles" between the dark matter and the disk (Bullock et al. 2001; van den Bosch 2002a; Maller \& Dekel 2002) and the inability of models to simultaneously match characteristics including the slope and zero-point of the T-F relation, slope and zero-point of the radius-luminosity relation, the luminosity function of spirals with reasonable values for the disk masses, halo structural parameters, and circular velocity relative to virial velocity (see, e.g., Dutton et al. 2006).

The build-up of angular momentum in rotating disks could be understood better through comparing theoretical models and simulations with observations of distant galaxies. In a first attempt at estimating the angular momenta of distant galaxies, Förster Schreiber et al. (2006) studied several $z \sim 2$ galaxies and found that, $\sim 10 \mathrm{Gyr}$ ago, galaxies appear to have approximately the same specific angular momentum as today's spirals, with values roughly similar to what is expected for their halos (see also Nesvadba et al. 2006). They argue that this confirms the hypothesis that baryons probably acquired their angular momentum during the collapse of their parent dark matter halos. However, as they pointed out, the spatial resolution of their observations does not allow them to distinguish between rotating disks and merger-induced kinematics, thus the origin of high angular momentum in $z \sim 2$ disk galaxies still remains unclear.

At lower redshift, a sample of $z \sim 0.6$ galaxies has been observed using the multi-integral field spectrograph FLAMES/GIRAFFE at VLT (see Flores et al. 2006; Puech et al. 2006, hereafter Papers I and II, respectively). GIRAFFE observations are confronted with the same difficulties in terms of spatial resolution as any other study of high redshift galaxy dynamics. However, to mitigate these effects, we developed a classification scheme based on the kinematics and morphologies of the galaxies, separating them into rotating disks and galaxies with complex or disturbed kinematics and morphologies (see Paper I for details). Given all of the other comparisons made in subsequent papers, e.g., Paper II and Puech et al. (2006b, hereafter Paper III), this classification method appears very robust (see detailed discussions in both Papers I and II). The goal of this paper is to derive the specific angular momentum in these $z \sim 0.6$ galaxies. The kinematics and emission line properties of this sample have been studied in detail in the three previous papers of this series (dedicated to the GIRAFFE Guaranteed Time Observation sample; see Papers I, II, and III). This paper is the fourth of this series and is organized as follows: Sect. 2 summarizes the observations and introduce the methodology, Sect. 3 presents the specific angular momentum of the GIRAFFE sample, Sect. 4 discusses the dynamical support of distant rotating disks, while Sects. 5 and 6 discuss the implications and summarize our results.

\section{Observations and methodology}

As part of the Guaranteed Time Observations (GTO) of the Paris Observatory, we did observations with the multi-integral field spectrograph FLAMES/GIRAFFE of a complete sample of 32 galaxies, with redshifts ranging from 0.4 to $0.7, E W_{\mathrm{o}}(\mathrm{OII}) \geq$ $15 \AA$, and $I_{\mathrm{AB}} \leq 22.5$. Briefly, we used the LR04 and LR05 grating, changing the setups to specifically target the [OII] $\lambda \lambda 3726$, 3729 doublet (with $R \sim 10000$ ); integration times ranged from 8 to 13 hours; the seeing was typically $\sim 0.6-0.7$ arcsec during all the observations. Data cubes were reduced using the GIRBLDRS v1.12 package (Blecha et al. 2000), including a flatfielding. Sky was carefully subtracted with our own IDL procedures. We derived both velocity fields and velocity dispersion maps for these galaxies in Papers I and II. These maps, as well as HST morphology, were used to divide the sample into three distinct classes based mainly on their dynamical characteristics: rotating disks, perturbed rotators, and galaxies with complex kinematics. A complete description of the GTO sample and of the methods and analysis used to classify the galaxies from our sample are given in Paper I.

The specific angular momentum $j$ of a rotating system can be estimated as (see, e.g., Mo et al. 1998)

$j=\beta R V_{\max }$, 


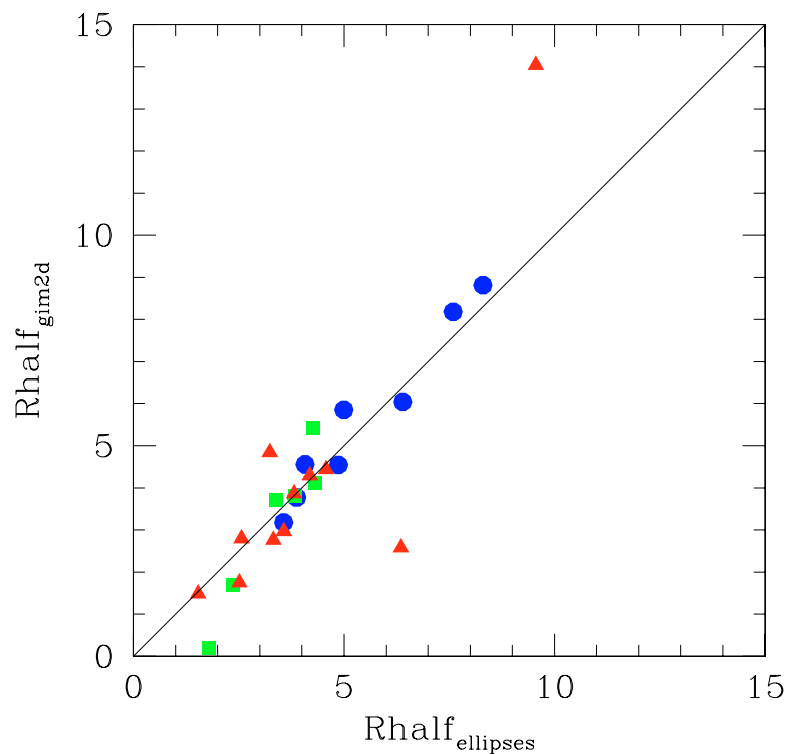

Fig. 1. Comparison of half-light radii (in kpc) derived using GIM2D and using the modeled results from fitting isophotal ellipses to the galaxy light profile. The resulting half light radii derived from both methods agree very well, where the galaxies with disturbed or complex kinematics show additional scatter. Blue dots represent galaxies classified as rotating disks, green squares represent perturbed rotations, and red triangles represent galaxies with complex kinematics.

where $\beta$ is a dimensionless parameter that depends on the geometry and spatial distribution of mass, $R$ a characteristic radius of the mass distribution, and $V_{\max }$ the maximal rotation velocity. For a thin exponential disk of scale length $R_{\mathrm{d}}$, this relation becomes

$j_{\text {disk }}=2 R_{\mathrm{d}} V_{\max }$.

To estimate a disk scale length, $R_{\mathrm{d}}$, it is necessary to deconvolve the disk from the bulge component. We used GIM2D (Simard \& Pritchet 1998; Simard et al. 2002) to measure $R_{\mathrm{d}}$ in the galaxies observed with GIRAFFE using HST/WFPC2 images $\left(0.1\right.$ arcsec pixel $\left.^{-1}\right)$ or, preferably, ACS images $\left(0.05\right.$ arcsec pixel $\left.^{-1}\right)$ when they were available. In Papers I and II, we derived the half-light radius from the modeling of isophotal ellipses (see also Hammer et al. 2001). Figure 1 shows that both half-light radii derived using this method and GIM2D agree very well. Unfortunately, GIM2D does not estimate any uncertainty for the half-light radius, but it is noteworthy that a linear fit, between the half-light radii derived using GIM2D and the modeling of isophots, returns a median standard deviation of $\sim 0.29 \mathrm{kpc}$, in close agreement with the typical uncertainty of $\sim 0.34 \mathrm{kpc}$ on the half-light radius derived from the isophotal ellipses modeling, as claimed in Paper I. Given that the fitting methods are independent, this comparison suggests that our $R_{\mathrm{d}}$ measurements using GIM2D are robust. Uncertainties on $R_{\mathrm{d}}$ were directly taken as 1 -sigma uncertainties returned by GIM2D, with a median value of $0.12 \mathrm{kpc}$.

Unfortunately, for five galaxies, we only had groundbased images available that were taken at the CFHT (0.207 arcsec pixel ${ }^{-1}$; see, e.g., Schade et al. 1996). Because these images are significantly affected by the relatively large seeing disk (large compared to the $R_{\mathrm{d}}$ of the disks), we did not attempt to use GIM2D to fit their disk light profiles since the solution would certainly be highly degenerate and thus unlikely to be reliable. Instead, we used the fact that for an exponential disk, $R_{\mathrm{d}}$ can theoretically be obtained from the half light radius $r_{\text {half }}$

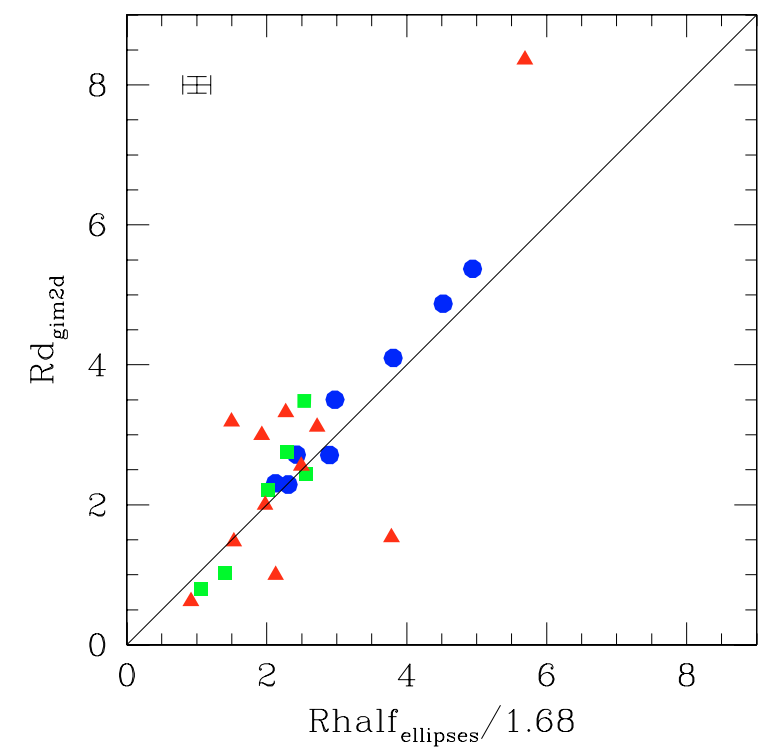

Fig. 2. Comparison of disk scale lengths (in kpc) derived using GIM2D and using the modeled results from fitting isophotal ellipses to the galaxy light profile. The resulting disk scale length from both methods agree very well, where the galaxies with disturbed or complex kinematics show additional scatter. Blue dots represent galaxies classified as rotating disks, green squares represent perturbed rotations, and red triangles represent galaxies with a complex kinematics. The median uncertainties, i.e., $0.12 \mathrm{kpc}$ for GIM2D and $0.34 / 1.68$ for the modeling of the isophots, are indicated in the upper-left corner.

using $R_{\mathrm{d}}=r_{\text {half }} / 1.68$. In Fig. 2, we compared $R_{\mathrm{d}}$ as measured by GIM2D with $r_{\text {half }} / 1.68$ as deduced from Papers I and II. This figure shows that this is a reasonable assumption (at least for rotating quiescent disk galaxies). We also used this method for two galaxies for which GIM2D failed to provide a statistically robust fit to the light profiles.

Both Figs. 1 and 2 show that for galaxies exhibiting more complex kinematics than a simple rotating disk (i.e., those classified as perturbed rotation or complex), the estimates of $R_{\mathrm{d}}$ and/or $r_{\text {half }}$ have a larger relative dispersion. In general, however, even the galaxies with complex kinematics have $R_{\mathrm{d}}$ and/or $r_{\text {half }}$ consistent with the average results of the rotating disks. The relatively large dispersion can be easily understood as a result of their unrelaxed dynamical state: it is likely that these galaxies are not in rotational equilibrium, and an exponential disk model is then probably an inadequate representation of their true light profile. However, to get homogeneous and consistent estimates, we chose to treat the whole sample, whatever the kinematical class, as if all galaxies were rotating disks. We also note that, despite the increased scatter between the various estimates of the scale length and half-light radius, each kinematic subsample generally falls along the one-to-one line. It is simply that the individual estimates are less reliable for the galaxies with complex kinematics but not for the ensemble of each class.

The maximal circular velocity $V_{\max }$ was estimated by fitting a double Gaussian to the [OII] doublet (see Paper I). This introduces a random uncertainty on the measurement of $V_{\max }$ that is estimated to be $\sim 10 \mathrm{~km} \mathrm{~s}^{-1}$, from the comparison of independent fits to the data. This uncertainty is relatively low because of the high spectral resolution of GIRAFFE $(R \sim 10000)$, which allows a good velocity measurement. Moreover, the limited size of the IFU can introduce an observational source of uncertainty, if this size is too small to reach the flat part of rotation curves. However, we showed in Paper I that the size of the GIRAFFE 
IFU is well-suited to measuring $V_{\max }$ for all the galaxies of the sample.

Because of the coarse sampling of the GIRAFFE IFU data (pixel size of 0.52 arcsec, $\sim 3.5 \mathrm{kpc}$ at $z \sim 0.6$ ), a mean correction of $20 \%$ has to be applied to $V_{\max }$. This correction factor is appropriate for $z \sim 0.6$ rotating disks (see discussion of this in Paper I). This correction factor was determined by simulating GIRAFFE observations from hydrodynamical simulations of a Sbc-like rotating disk. As explained in Papers I and II, we are probably severely underestimating this correction factor for objects showing more complex kinematics (see Paper II), and this could introduce a relative offset between rotating disks and objects with more complex kinematics. The absolute uncertainty of the correction factor for rotating disks was determined to be $\pm 4 \%$ because of possible variations in size and inclination (see Paper I). An additional source of uncertainty on this correction factor could be due to variations in the dynamical properties along, e.g., the Hubble sequence. We checked that, by using another hydrodynamical simulation of a "mean" SDSS galaxy, a mean $20 \%$ correction factor is still appropriate, within $\pm \sim 4 \%$ relative to a $20 \%$ correction factor. We emphasize that this uncertainty is very difficult to estimate, given the high number of parameters that have to be taken into account.

Finally, uncertainties on $j_{\text {disk }}$ were estimated using the usual methods, i.e. propagating the individual uncertainties on $V_{\max }$, $R_{\mathrm{d}}$, and on the correction factor on $V_{\max }$, as explained above. This gives a median 1-sigma uncertainty of $0.09 \mathrm{dex}$ and 0.10 dex in $V_{\max }$ and $j_{\text {disk. }}$. This is to be compared with typical uncertainties for local galaxies of 0.03 and 0.06 dex respectively, as estimated from Courteau (1997). We emphasize that such an uncertainty is meaningful only for rotating disks, since the accuracy and appropriateness of the model used to reproduce the data is not taken into account. This uncertainty should then be viewed as a random uncertainty associated with $j_{\text {disk }}$, if the rotating disk model is correct, but certainly does not include the systematic uncertainty when such a model is not appropriate. Indeed, in Papers I and II, we emphasized that objects showing complex kinematics are probably mergers or merger remnants. For these objects, the $j_{\text {disk }}$ derived as above should then be viewed as the orbital angular momentum due to the relative motion of the two progenitors, rather than the intrinsic spin angular momentum of a single rotating disk (see also Förster Schreiber et al. 2006). We discuss this in more detail subsequently.

\section{Angular momentum of distant galaxies}

To compare distant with local galaxies, we used two local samples from Courteau (1997) and Mathewson et al. (1992). For the first sample, we used the compilation made by Steinmetz \& Navarro (1999) and kindly provided by M. Steinmetz. For the second sample, we retrieved the electronically available data from the $\mathrm{CDS}^{1}$. For this sample, no $R_{\mathrm{d}}$ measurement was directly available, so we derived it from their ( $I$ band) 23.5 magnitude isophotal radius by dividing by a mean correction factor of 3.5 (Palunas \& Williams 2000).

Figure 3 shows the disk scale length versus the maximal rotation velocity, both for local and distant samples. Both local samples agree quite well, in spite of the different proxies used for the maximal rotational velocity and the disk scale length. Distant rotating disks fall close to local rotating disks, although some of them appear to have a relatively lower disk scale length. Those are associated to galaxies with a half-light radius slightly lower

\footnotetext{
1 http://cdsweb.u-strasbg.fr/
}

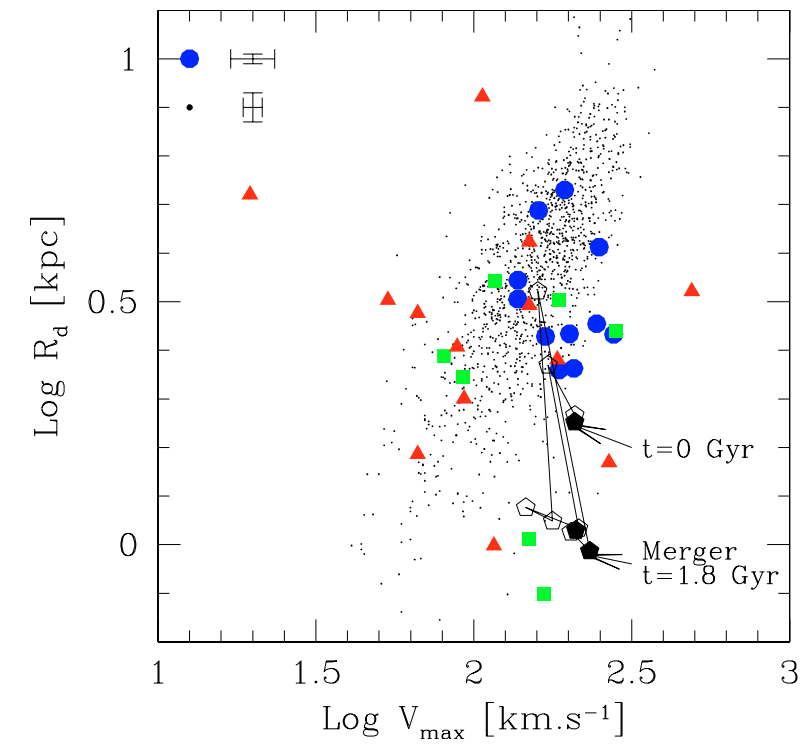

Fig. 3. Disk scale length versus maximal rotational velocity, for the sample of 32 distant galaxies presented in Papers I and II. Blue dots represent galaxies classified as rotating disks, green squares those classified as perturbed rotators, and red triangles galaxies with complex kinematics. The small black dots represent the local sample of Courteau (1997) and Mathewson et al. (1992). The median 1-sigma uncertainties are indicated in the upper-left corner for both distant rotating disks (blue dots) and local disks (black dots). For local galaxies, $R_{\mathrm{d}}$ have been determined using 1D fits, whereas 2D fits were used for distant galaxies: this explains why the uncertainty along the ordinate is greater for local than for distant galaxies. Black pentagons represent simulated GIRAFFE observations using a hydrodynamical simulation of a major merger of two Sbc galaxies. The beginning and the end of the sequence, as well as the merger itself, are indicated by solid symbols.

than that of compact galaxies (i.e., $R_{\text {half }} \leq 4.7 \mathrm{kpc}$, see Paper II). On the other hand, more kinematically disturbed distant galaxies show a very high dispersion around the local $R_{\mathrm{d}}-V_{\max }$ relation. It can be explained in two ways. First, more kinematically complex distant galaxies have larger uncertainty in determination of their radius. This uncertainty can be estimated from the scatter of kinematically complex galaxies in Fig. 2 and is $\sigma \sim 1.45 \mathrm{kpc}$. Relative to the median $R_{\mathrm{d}}$ of kinematically complex galaxies, this translates into a 0.2 dex scatter in Fig. 3. It is thus clear that this is not sufficient to explain the extremely large scatter of the kinematically complex galaxies in the $R_{\mathrm{d}}-V_{\max }$ plane (i.e., $\sigma \sim 0.45$ dex relative to the fit of the local galaxies $R_{\mathrm{d}}-V_{\max }$ relation). The second (and most important) source of scatter is that rotational velocities undergo abrupt spatial and temporal variations during dynamical processes such as minor and/or major mergers. This effect is illustrated by the track that represents a hydrodynamical simulation of a merger of two Sbc galaxies from Cox et al. (2006). GIRAFFE observations were simulated along the merging sequence, as well as HST images (Puech et al., in preparation). From these simulated data, both rotation velocities and disk scale lengths were extracted and corrected following similar methods to those used for real distant galaxies (see previous section). During this simulation, the merger occurs at $\sim 1.8 \mathrm{Gyr}$ after the beginning of the simulation and is simulated for up to 0.5 Gyrs after the merger. At the end of the simulation, the remnant looks relatively similar to an elliptical, with a small inner disk at the center. It is beyond the scope of this paper to explore it for every possible track in Fig. 3, varying, e.g., the gas fraction, orbital parameters, or the amount of feedback. What is important to note here is that this simulation, which includes 


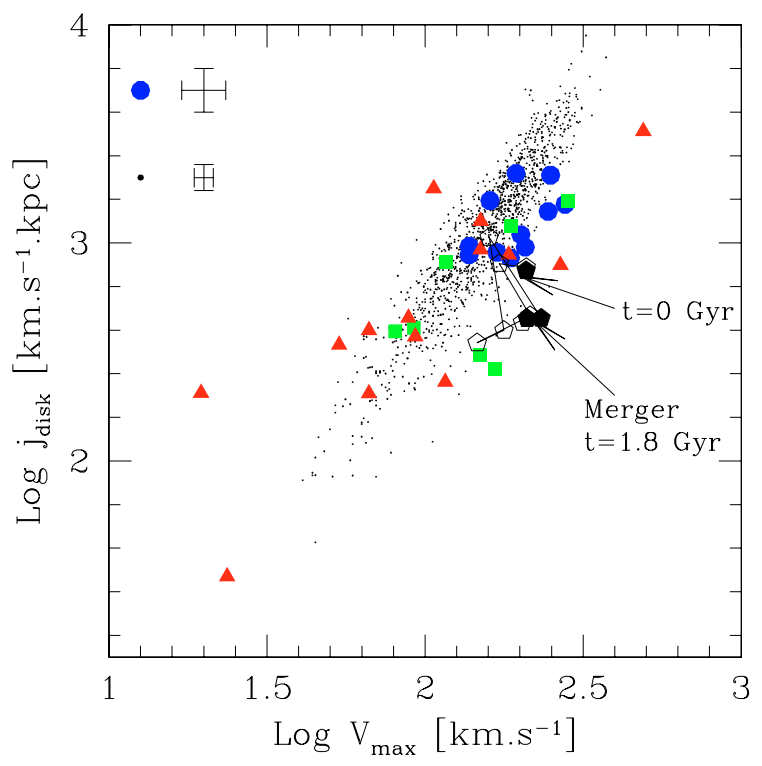

Fig. 4. Specific angular momentum of the disk, $j_{\text {disk }}$, versus their maximum rotation velocity, $V_{\max }$, for the sample of 32 distant galaxies presented in Papers I and II. Blue dots represent galaxies classified as rotating disks, green squares those classified as perturbed rotators, and red triangles galaxies with complex kinematics. The small black dots represent the local sample of Courteau (1997) and Mathewson et al. (1992). The median 1-sigma uncertainties are indicated in the upper-left corner for both distant rotating disks (blue dots) and local disks (black dots). Black pentagons represent simulated GIRAFFE observations using a hydrodynamical simulation of a major merger of two Sbc galaxies. The beginning and the end of the sequence, as well as the merger itself, are indicated by solid symbols.

possible observational or instrumental biases, illustrates how the scatter of the most kinematically disturbed distant galaxies could be easily reproduced by such events. This strengthens the idea that these objects are probably ongoing merger and/or merger remnants.

Figure 4 shows the specific angular momentum of the rotating disks in the sample of galaxies observed with GIRAFFE, $j_{\text {disk }}$, versus their maximum rotation velocity, $V_{\max }$. In this figure, $z \sim 0.6$ rotating disks show a specific angular momentum comparable to that of local galaxies with same the $V_{\max }$ (i.e., $\sim$ same total mass). Objects with a complex kinematics have a lower median specific angular momentum, with $\log j_{\text {disk }} \sim 2.7$, than in rotating disks, which have a median of $\log j_{\text {disk }} \sim 3.0$. This is probably again due to the fact that $V_{\max }$ can be severely underestimated for galaxies with complex kinematics (see Papers I and II, and above): their derived specific angular momentum should then be considered as lower bounds. As in Fig. 3, the dispersion of distant galaxies with complex kinematics is significantly higher. As these objects are hypothesized to be undergoing a merger or are merger remnants, we are likely to be measuring an orbital angular momentum rather than an intrinsic spin angular momentum of a single disk. In such a case, the shape factor $\beta=2$ is probably not appropriate and could be an source of additional dispersion. To investigate this effect, we also over-plotted the same simulation of GIRAFFE observations of a merger of Sbc galaxies in this figure, as described above. Note that the beginning of the sequence corresponds to only one of the two progenitors, which shows a deficit in specific angular momentum compared to local galaxies. This could be related to the difficulty for hydrodynamical simulations to reproduce the higher angular momentum observed in local disks. The position of most galaxies with complex kinematics in the $j_{\text {disk }}-V_{\max }$ plane can here again be easily explained as a result of mergers. As these simulated data include many possible sources of uncertainty, it clearly suggests that the larger scatter of galaxies showing complex kinematics can be associated with mergers or merger remnants.

\section{Dynamical support of distant disk galaxies}

In this section, we compare the dynamical support (rotation vs. velocity dispersion) of distant and local rotating disks. Such an analysis could help us to understand how rotating disks acquire and then lose their angular momentum.

The most revealing quantity for estimating the dynamical support of galaxies is the ratio of rotation velocity to velocity dispersion of the disk, $V / \sigma$. Here, $V$ is a circular velocity (quantifying the amount of rotation), and $\sigma$ is an estimate of the intrinsic velocity dispersion in the disk (i.e., turbulent or peculiar motions). In elliptical galaxies, $V / \sigma$ is usually estimated via the ratio of the maximal rotational velocity to the mean velocity dispersion within $0.5 r_{\mathrm{e}}$, where $r_{\mathrm{e}}$ is the effective radius of the system (e.g., Davies et al. 1983; Bender et al. 1994). For spiral galaxies, there is no general consensus: different spatial components of $\sigma$, estimated following different methods, have been used (e.g., Bottema 1993; Vega Beltrán et al. 2001; Hunter et al. 2005). Binney (2005) demonstrates that with integral field spectroscopy (i.e., a 2-dimensional spatial coverage combined with simultaneous spectral coverage), the intrinsic $V / \sigma$ is more robustly estimated using the ratio between the mean squared rotational velocity and the mean squared velocity dispersion, both measured directly along the line of sight.

We chose to estimate $V$ using the maximal rotational velocity (corrected for the inclination and spatial resolution effects, see Sect. 2), as it is probably the most accurate quantity derivable from GIRAFFE velocity fields. Because the velocity gradient of the rotation curve of $z \sim 0.6$ rotating disk galaxies falls approximatively in only one GIRAFFE IFU pixel (or about one resolution element given the seeing, see Sect. 2), the center of the GIRAFFE velocity dispersion maps show a peak that is due to shear and/or large-scale motions in velocity so cannot be used to estimate the intrinsic velocity dispersion of the disk (see Papers I and II). On the contrary, the regions surrounding the peak of the velocity dispersion are much less affected by the shear and/or large-scale motions in velocity, since the rotation curve is approximatively flat in these regions (i.e., has a constant velocity with radius). These outer regions of the velocity dispersion maps can then be used to construct a reliable estimate of the intrinsic velocity dispersion of the disk. To construct such reliable estimates of the intrinsic velocity dispersion of the disks, we first removed the $\sigma$ peak due to rotation in the velocity dispersion map, guided by the modeling of the dispersion we made in Paper I. We then estimated $\sigma$ by deriving the signal-to-noise weighted mean of the remaining pixels.

Unfortunately, there are very few published velocity dispersion maps of local galaxies that could be used for direct comparison. However, several dozen velocity dispersion profiles of the gas in local disks that were obtained with long-slit spectroscopy have been published. We combined the samples of Vega Beltrán et al. (2001), Corsini et al. (2003), and Pizzella et al. (2004), which are composed of spiral galaxies with an earlier morphological type than Sc. Note that $V / \sigma$ ratio does not seem to depend on the morphological type, since a similar range of values is found for both spiral and irregular galaxies (see Hunter et al. 2005). We checked that both local and distant 
samples have similar distributions in the absolute $B$ magnitude, $M_{B}$, and we kept only galaxies with an absolute $B$ magnitude brighter than the lower value found in the GIRAFFE sample (i.e., $M_{B}(A B) \leq-19.26$ ), to ensure that both samples are probing galaxies with comparable stellar masses and star-formation rates. To exclude the central dynamically hot region, we performed a sigma clipping on the velocity dispersion profile of local galaxies, keeping only the points along the curve that were below $2 \sigma$ around the median. We then took the final median of the remaining points as a measure of $\sigma$ in the disk.

If measured when projected onto inclined disks, the observed velocity dispersion is a combination of the three spatial components of the velocity dispersion - the radial component $\sigma_{r}$, the azimuthal component $\sigma_{\phi}$, and the vertical component $\sigma_{z}$ (e.g., Binney \& Merrifield 1989):

$\sigma_{\mathrm{obs}}^{2}=\left(\sigma_{r}^{2} \sin ^{2} \eta+\sigma_{\phi}^{2} \cos ^{2} \eta\right) \sin ^{2} i+\sigma_{z}^{2} \cos ^{2} i$

where $\eta$ is the angle between the observed PA and the major axis of the galaxy, and $i$ the inclination angle of the disk. In local spiral galaxies, $\sigma_{z} \sim \sigma_{\phi} \sim 0.7 \sigma_{r}$ for stars (e.g., Hunter et al. 2005). In the case of local galaxies, if we assume both a well-aligned slit and well-coupled gas and stars dynamics (see Vega Beltrán et al. 2001; Pizzella et al. 2004), one gets $\sigma_{\mathrm{obs}} \sim \sigma_{z}$. In the case of distant galaxies, observed using integral field spectroscopy, it is necessary to correct for the different $\eta$ corresponding to the different IFU pixels. We can directly correct for this effect on the mean sigma by averaging the $\cos ^{2} \eta$ and $\sin ^{2} \eta$ term, and then multiplying by a $\left(1+\sin ^{2} i / 2\right)^{-1 / 2}$ correction factor. Finally, we obtain an estimate of the spatially averaged $\sigma_{z}$, which can be used in the $V / \sigma$ ratio. To check that the final estimate of $V / \sigma$ does not significantly depend on the method used to estimate $\sigma$, we compared with the velocity dispersion measured at $r_{\text {half }} / 4$ as given by Vega Beltrán et al. This alternative method does not significantly affect the general trend of $V / \sigma$ estimated in local disks.

In the sample of local galaxies, errors have been estimated as follows. The uncertainty on $V$ was directly taken as the mean of the velocity measurement uncertainty on $V_{\max }$ and $V_{\min }$ as given by Vega Beltrán et al. (2001), Corsini et al. (2003), and Pizzella et al. (2004). The uncertainty on $\sigma$ was estimated as the median of measurement uncertainties of the points of the velocity dispersion curve kept during the sigma clipping (see above). A conservative uncertainty of three degrees was assumed for the inclination. We found a median uncertainty of 1.6. Figure 5 shows how this uncertainty evolves with $V / \sigma$. This figure shows that high values of $V / \sigma$ have large associated uncertainties; including only sources with $V / \sigma$ estimates greater than three times their uncertainty left us with a range of local $V / \sigma$ values ranging between 2 and 9 , with a median uncertainty in $V / \sigma$ of 1.1 . In the following, we only consider these points, as others galaxies have $V / \sigma$ ratios that are too uncertain. For distant rotating disks, we estimated the uncertainty of $\sigma$ to be lower than $15 \mathrm{~km} \mathrm{~s}^{-1}$ (see Paper I). We added an additional uncertainty term due to the correction factor used to deconvolve $V_{\max }$ (see Sect. 2).

Figure 6 shows the comparison of $V / \sigma$ as a function of the ellipticity in both distant and local rotating disk galaxies. The median $V / \sigma$ is $3.8 \pm 2$ for distant rotating disks and $6.1 \pm 1.1$ for local disks. Recall that the highest local $V / \sigma$ have been discarded in this comparison because of an uncertainty too large: this could result in an underestimation of the local median $V / \sigma$. We also plotted the $V / \sigma$ ratio for the distant perturbed rotating disks, with a median of $2.4 \pm 2.5$. Figure 6 shows that distant disks have lower $V / \sigma$ ratios than local ones. This is consistent with the fact these galaxies are probably undergoing a minor merger

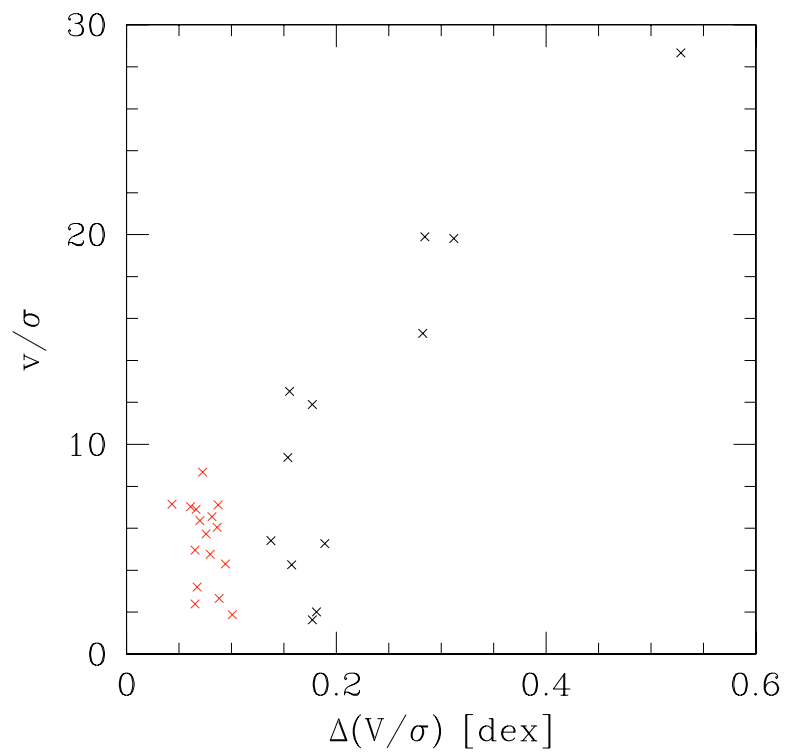

Fig. 5. $V / \sigma$ vs. uncertainty in $V / \sigma$ for the sample of local galaxies used for comparison. The galaxies with significant $V / \sigma$ estimates $(V / \sigma \geq 3 \Delta$ $(V / \sigma))$ are shown in red.

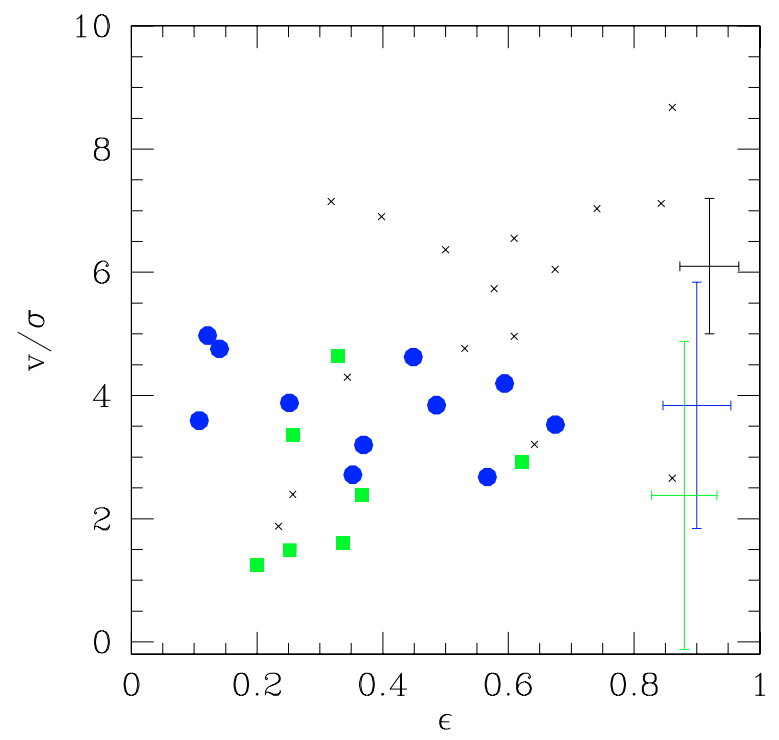

Fig. 6. Comparison of $V / \sigma$ and ellipticity, $\epsilon$, for galaxies at $z \sim 0.6$ compared to a matched sample of local galaxies. The $V_{\max } / \sigma$ ratio for galaxies at $z \sim 0.6$ that have been determined to be rotating disks (blue dots) and to have rotation curves that are perturbed (green squares) are compared with local galaxies whose morphology and velocity curves are consistent with rotating disks (black crosses). The medians are indicated by the position of the median uncertainties on the right side of the diagram (each represented by matching colors).

and/or a gas accretion event that is heating their disks (Walker et al. 1996; Velazquez \& White 1999; Abadi et al. 2003), as was suggested in Paper II. More data will be needed to confirm this trend, however.

\section{Discussion}

\subsection{The growth of angular momentum in galaxies with time}

We showed in Sect. 3 that the $z \sim 0.6$ and local disks of the same total mass show comparable specific angular momentum. More dynamically complex objects fall around the same $j_{\text {disk }}-V_{\max }$ 
relation but with much larger dispersion than the local relation. How can we interpret this in terms of angular momentum growth with time in galaxies?

One of the first theories proposed for the build up of angular momentum in galaxies is the tidal torque theory (Peebles 1969; White 1984). The basic idea in the tidal torque theory is that most of the angular momentum is being gradually acquired in the linear regime of growth of the density fluctuations, due to tidal torques from neighboring fluctuations. This process continues relatively efficiently until the halo reaches its turn around time, i.e., roughly when the protogalaxy decouples from the Hubble flow. Moreover, this theory suggests that angular momentum gain is minimal when halos are growing nonlinearly after they have decoupled from the expanding background and have formed virialized systems. This theory then predicts rapid growth of angular momentum in the early evolution of massive halos, and subsequently little growth (Porciani et al. 2002). At first sight, our results, when combined with those of Förster Schreiber et al. (2006) and Nesvadba et al. (2006), would mean that the specific angular momentum of rotating disks does not evolve with redshift from $z \sim 2$ to the present. This would indicate that this scenario for the growth of angular momentum is plausible. However, we have to caution that current samples are relatively small and incomplete in terms of galaxy mass. Moreover, there are some issues with the physical resolution of the data, especially at $z>2$ (but see Nesvadba et al. 2006). Even though these results are all formally consistent with no overall evolution in the specific angular momentum, given the limitations of all studies, it is difficult to conclude anything about evolutionary trends in the specific angular momentum in rotating disk galaxies.

Beyond this, the results presented here can constrain how angular momentum and indeed how galaxies grow after the epoch when massive halos (like the ones studies here) acquired their angular momenta through tidal torquing. The basic ideas that have emerged from theoretical research are that the specific angular momentum can be increased or decreased in a halo through a random-walk process of interactions and merging with other halos (e.g., Gardner 2001; Vitvitska et al. 2002; Porciani et al. 2002) or through gas accretion onto the halo (e.g., White \& Rees 1978). In the merger process, angular momentum is gained or lost depending on the geometry, dynamics, the detailed mass distribution of the merging halos, strength of the feedback, the relative masses of the merging halos and galaxies, etc. (e.g., Robertson et al. 2005; Springel \& Hernquist 2005; D’Onghia et al. 2006). Alternatively, the angular momentum of disk galaxies could grow through the accretion of cooling halo gas or smooth accretion of mass into the dark matter halo (e.g., White \& Rees 1978; Peirani et al. 2004; van den Bosch 2001, 2002a; van den Bosch et al. 2002b, 2003; Chen et al. 2003; Okamoto et al. 2005). The amount of angular momentum change then depends on the relative angular momentum of the gas and dark matter, the way the gas was accreted, the amount of mechanical dissipation the gas undergoes during collapse, the orientation of its angular momentum vector relative to the perhaps pre-existing disk, etc.

Returning to both Figs. 3 and 4 (see also the appendix), it is clear that perturbed rotation and kinematically complex galaxies are dynamically different from dynamically relaxed local disks. The dispersions in the $j_{\text {disk }}-V_{\max }$ are indeed similar for the rotating disks, higher for the perturbed rotators, and very high for the galaxies with complex dynamics. We also note that some of the distant galaxies that are not rotating disks have offsets to both lower and higher $j_{\text {disk }}$ relative to local galaxies for the same $V_{\max }$.
Because we are very likely underestimating $j_{\text {disk }}$ in kinematically complex galaxies (see Sect. 3.1), we cannot conclude anything specifical about any difference on median $j_{\text {disk }}$ between the different kinematical classes. However, the larger dispersion in the $V_{\max }-j_{\text {disk }}$ plane of kinematically complex galaxies relative to distant rotating disks is significant. This dispersion is caused by abrupt variations in the angular momentum in galaxies with complex kinematics and are probably the result of a randomwalk during a non-linear phase in the evolution of the angular momentum. This is the first observational evidence for such a non-linear growth of the angular momentum in galaxies, as expected from theoretical models (e.g., Gardner 2001; Vitvitska et al. 2002; Peirani et al. 2004).

An important question is what the driving mechanism of this random walk is. Theoretical models show that major mergers cause the most abrupt variations in the angular momentum, while minor mergers and/or gas accretion are associated with smoother variations (e.g., Vitvitska et al. 2002; Peirani et al. 2004; Hetznecker \& Burkert 2006). If the gas is heated to approximately the virial temperature of the halo before collapsing onto, or to form, a pre-existing disk (Dekel \& Birnboim 2006), we would expect the gas to have a specific angular momentum similar to or greater than that of the dark matter halo (e.g., Chen et al. 2003; Okamoto et al. 2005). This is why we might then expect the specific angular momentum of the disk to remain constant or to mildly increase or decrease at late times (Peirani et al. 2004). It is hard to believe that smooth gas accretion alone could explain the complex or perturbed kinematics and/or discrepant values of the specific angular momentum. We instead suggest that mergers (both major and minor mergers, possibly associated with complex kinematic galaxies and perturbed rotations, respectively, see Papers I and II) play a significant role in changing the angular momentum of galaxies with time. While overall consistent with the tidal torque theory, the increased dispersion in specific angular momentum and spin of the perturbed rotators and galaxies with complex kinematics is consistent with the merger scenario. Vitvitska et al. (2002) show that within the context of merging, we would find both increases and decreases in the specific angular momentum, resulting in an increase in the dispersion of their angular momenta. This is consistent with what we observe when we compare distant rotating disks to galaxies with complex kinematics. This interpretation is strongly supported by a simulation of GIRAFFE observations of a major merger. This simulation illustrates how the higher dispersion of kinematically complex galaxies can arise from mergers, taking all possible observational uncertainties into account.

\subsection{Building local disk galaxies}

In the following discussion, we assume that most of the distant galaxies observed with emission lines are progenitors of local disks. This should be the case even for most galaxies showing complex kinematics and perturbed rotations. Indeed, those galaxies represent $40 \%$ of galaxies at $z \sim 0.6$; and if they were $\mathrm{E} / \mathrm{S} 0$ progenitors, there would be a much higher fraction of E/S0 than is observed today (see, e.g., Hammer et al. 2005; Lotz et al. 2006).

Because we are comparing samples of galaxies spanning different total masses $\left(V_{\max }\right)$, linking distant-to-local disks from Fig. 4 alone is not straightforward. Can $z \sim 0.6$ galaxies evolve towards local disks through major mergers? Hammer et al. (2005) claim that $75 \%$ of local spiral in the $10^{10.5}-10^{11.5} M_{\odot}$ range (the so-called "intermediate-mass" range, see also Hammer et al. 2006) have experienced a major 
merger since $z=1$. Assuming an evolution rate as $(1+z)^{2.7}$ (Le Fèvre et al. 2000), one can derive that $29 \%$ of local spiral galaxies have experienced a major merger since $z=0.6$. Similarly, Lotz et al. (2006) estimate that between $33 \%$ and $66 \%$ of $L_{B} \geq 0.4 L_{B}^{*}$ galaxies had a major merger since $z=1.1$, with an evolution rate of $(1+z)^{1.12}$. Combining the above estimates implies that $\sim 15$ to $30 \%$ of local spirals could have experienced a major merger since $z=0.6$. Then, the majority of $z \sim 0.6$ rotating disks cannot evolve towards local spirals through major mergers. Another possibility is that $z \sim 0.6$ galaxies could have evolved to $z=0$ spirals through minor mergers and/or gas accretion: their rotating disks would then have survived, and their specific angular momentum should statistically increase by only $\sim 0.1$ dex (Peirani et al. 2004). A third possibility is that distant disks could evolve in a "closed box". In the absence of external torques, their specific angular momentum would then remain constant. Finally, the last two possible evolution tracks from $z \sim 0.6$ to $z=0$ are equally viable.

This picture is also supported by the fact that distant disks (we consider here both rotating disks and perturbed rotating disks together) seem to be heated compared to local disks (i.e., have systematically higher $V / \sigma$ ), possibly through minor mergers and/or gas accretion (Walker et al. 1996; Velazquez \& White 1999; Abadi et al. 2003). A difficulty in quantifying the uncertainty in this result could arise from the different observational strategies used in the local and distant samples (slit vs. integral field spectroscopy, respectively). This, however, is unlikely given the fact that most spirals today are strongly dominated by rotational motions (e.g., Binney \& Merrifield 1989). An interesting additional component of the evolution in $V_{\max } / \sigma$ we observed is the possibility of "angular momentum mixing" during minor mergers and/or gas accretion events (see Okamoto et al. 2005). During such events, the direction of the angular momentum vector can change, and newly accreted gas may then settle into a different orientation from the pre-existing disk. During this process, the observational manifestations are likely to be relatively high velocity dispersion of the gas caused by the gas being shock-heated by the turbulence generated by the overlapping and dynamically different disks (what we could observe in Fig. 6).

Given the above discussion (see Sect. 5.1) about the specific angular momentum, it is unlikely that (smooth) gas accretion alone plays a large role in the general growth of rotating disks. It is also equally unlikely that gas accretion alone could explain the disturbed kinematics of perturbed rotation, as this process is expected to be dynamically smooth (see Papers I, II) and to only lead to small changes in the angular momentum. Note, however, that, to our knowledge, there is no clear theoretical prediction about the kinematics of gas accretion by galaxies. Thus, our results show that it is likely that mergers (and, a priori, both major and minor mergers) play an important role in the general buildup of rotating disks. The link between dynamical processes, such as mergers, and the dynamical classifications adopted in this series of paper will be addressed in a forthcoming article.

The results presented in this paper fit quite nicely within the "spiral rebuilding" scenario proposed by Hammer et al. (2005), where $\sim 75 \pm 25 \%$ of local spirals (those of early type) have undergone a merger since $z=1$ and have rebuilt a disk thanks to gas accretion. This scenario is composed of 3 major phases: a "pre-merger phase" during which two distant spirals merge, the "LCG phase" where all material from the progenitors fall into the mass barycenter of the system and form a bulge, and the "disk growing phase" where subsequently accreted material forms a rotating disk (see Fig. 8 of Okamoto et al. 2005, for how this might look). As the two progenitors are merging, their disks, and thus their spin angular momentum, are destroyed during the collision (Cox et al. 2004). At the same time, the encounter has a significant quantity of orbital angular momentum that can be progressively converted into spin angular momentum as a new disk is re-built around the remnant. Moreover, we saw that the increase in $V / \sigma$ in disk galaxies could be due to heating mechanisms such as minor mergers and gas accretion events, as if some of the disk rebuilding were triggered by accretion of the gas left over from the merging event. Yoachim \& Dalcanton (2006) suggest that the properties of thick and thin disks are consistent with gas-rich mergers playing a significant role in their formation where the stars in these mergers formed the thick disk, while the settling gas formed much of the thin disk. The results presented here amplify these ideas and show their credibility in directly explaining the dynamics of intermediate redshift disk galaxies.

\section{Conclusions}

We have studied the angular momentum and the dynamical support of a sample of $z \sim 0.6$ galaxies observed with the integral field spectrograph FLAMES/GIRAFFE. We found that the classification of distant galaxies based on their kinematic properties (mainly) and morphologies into three distinct classes (rotating disks, perturbed rotators, and kinematically complex galaxies) is apparently robust. This classification also appears to select galaxies with angular momenta that are consistent with local spiral galaxies but that show varying degrees of dispersion relative to the local values, increasing from rotating disks to kinematically complex galaxies.

This can be interpreted as evidence of a non-linear randomwalk evolution of the angular momentum during the past $8 \mathrm{Gyr}$. A natural driver for this random-walk is provided by major mergers, since both the dispersion of kinematically complex galaxies in the $j_{\text {disk }}-V_{\max }$ plane and the complexity of their velocity fields (see Puech et al. 2007, in preparation) can be reproduced by hydrodynamical simulations of these events. Major mergers could also explain how the angular momentum of local rotating disks could acquired and be as high as observed, namely through the conversion of orbital angular momentum to spin momentum. Moreover, distant disks appear to be more turbulent (lower $V / \sigma)$. This is likely indication that local disks could grow through the accretion of gas through mergers or in discrete clouds. In a refinement of this general picture, the higher velocity dispersions could be due to "angular momentum mixing" (see Okamoto et al. 2005) whereby the relative orbit of the infalling gas is skewed compared to the previous disk. The torque provided by the accreting gas causes a change in the angular momentum vector, thereby increasing the dispersion observed in the gas.

Finally, these findings are consistent with the observational scenario proposed by Hammer et al. (2005), where $\sim 75 \%$ of local spirals (those of early type) have undergone a major merger since $z=1$ and have then rebuilt their disks thanks to gas accretion (possibly from high angular momentum gas left over from the merging event) and/or minor mergers. Unfortunately, drawing robust conclusions as to the physical processes driving the observational manifestations we have discussed in this paper is difficult given the small numbers of galaxies we have studied. To increase the statistical robustness of these results, we are currently analyzing similar data for a much larger sample of several hundred disk galaxies as part of the VLT Large Program IMAGES (P.I.: F. Hammer). The galaxies are selected by 
absolute $J$-band magnitude to have redshifts of or less than 0.9 and to have $[\mathrm{OII}]$ equivalent width comparable to the galaxies studied here (Ravikumar et al. 2006). With the robust selection and large numbers, we should be able to make more definitive statements about the physical processes behind the dynamical and morphological evolution of spiral galaxies over the past 7 Gyrs.

Acknowledgements. We would like to thank M. Steinmetz for having provided us with their compilation of data on the kinematics of local galaxies and for interesting comments on recent simulation work. We are especially indebted to T. J. Cox who provided us with hydrodynamical simulations of a Sbc galaxy. We also would like to thank A. Dekel, C. Balkowski, P. Amram, and L. Chemin for very interesting discussions we had together about the subject of this paper.

\section{Appendix A: Spin parameter of the halos}

This appendix presents a perhaps more predictive comparison between local and distant galaxies' angular momentum, based on the use of the spin parameter of the surrounding halos, which has the advantage of being approximately independent of the halo mass (Barnes \& Efstathiou 1987; Lemson \& Kauffmann 1999; Maller \& Dekel 2002; Peirani et al. 2004). This dimension-less parameter measures the ratio between the true angular momentum of the system and the angular momentum it would have, if all the mass was entirely supported by rotation, or, equivalently, it measures the ratio of the spin energy to the total binding energy. The spin parameter (Peebles 1969) is given by:

$\lambda=\frac{J|E|^{1 / 2}}{G M^{5 / 2}}$,

where $J$ is the true halo angular momentum, $E$ the total energy, and $M$ the total mass of the system. It is possible to estimate $\lambda$ using the observed characteristics of the disk. Following the method outlined by Hernandez $\&$ Cervantes-Sodi (2006), $\lambda$ can be estimated using:

$\lambda \sim R_{\mathrm{d}} / V_{\mathrm{d}}^{2}$.

This formulation requires the following assumptions: (1) $j_{\text {disk }} \sim$ $j_{\text {halo }}$ (see discussion in the introduction); (2) the total disk mass must be a constant fraction of the mass of the halo; (3) the total energy is dominated by the halo which is virialized; (4) a baryonic Tully-Fisher relation exists that can be expressed by $M_{\mathrm{d}} \sim V_{\mathrm{d}}^{4}$ (see McGaugh 2005) $)^{2}$. For our analysis to be valid we also require that these assumptions are not dependent on the epoch of observation. In Fig. A.1, we plot the histogram of $\lambda$ in the local sample used in this paper. The line at $\lambda \sim 0.03$ is the most probable local value found by Tonini et al. (2006) using an independent method. It is very close to the peak of our histogram: in spite of its crudeness, this suggests that the method of estimating $\lambda$ is appropriate, at least for local disks.

In Fig. A.2, we used this relation to investigate the difference in the spin parameter, $\lambda$, between local and distant galaxies. From Peirani et al. (2004), the median $\lambda$ between $z=0.6$ and $z=0$ is expected to remain roughly constant with accretion alone, whereas an increase of $\sim 10 \%$ is expected, taking major merger events into account. Figure A.2 seems to favor a small increase in $\lambda$ from $z \sim 0.6$ to $z=0$. Unfortunately, the uncertainty on the median $\lambda$ of the $z \sim 0.6$ galaxies is very high, see Fig. A.2: it is then impossible to identify the relative contribution

${ }^{2}$ Hernandez \& Cervantes-Sodi (2006) use a baryonic Tully-Fisher relation slightly different from the one used in this paper, leading them to $\lambda \sim R_{\mathrm{d}} / V_{\mathrm{d}}^{3.5}$.

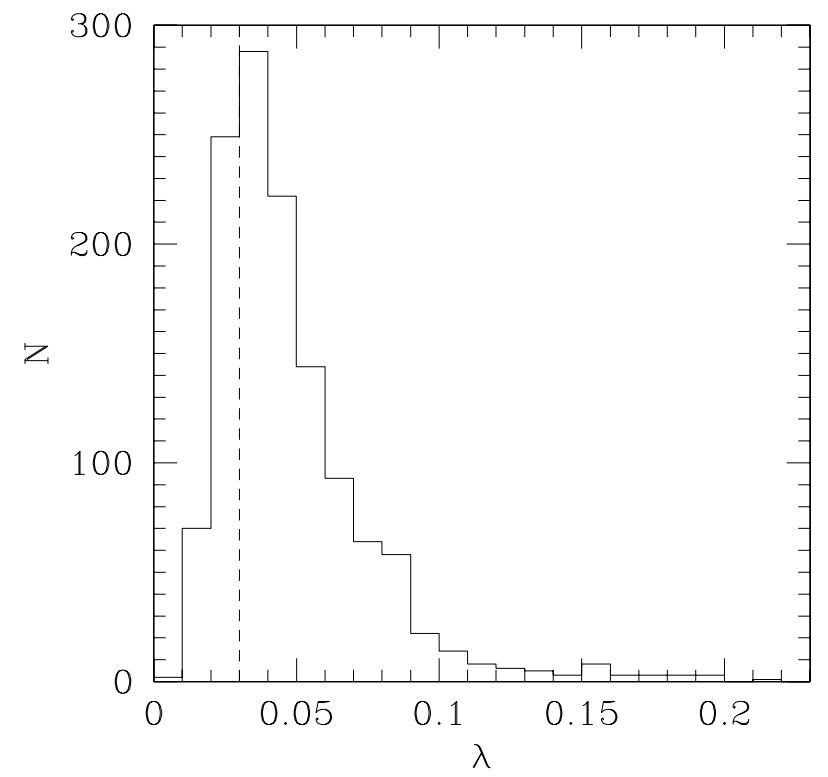

Fig. A.1. Histogram of $\lambda$ derived using the method outlined in the text for the local sample of galaxies. The most probable value is very close to the estimate made by Tonini et al. (2006) also using a local sample of galaxies.

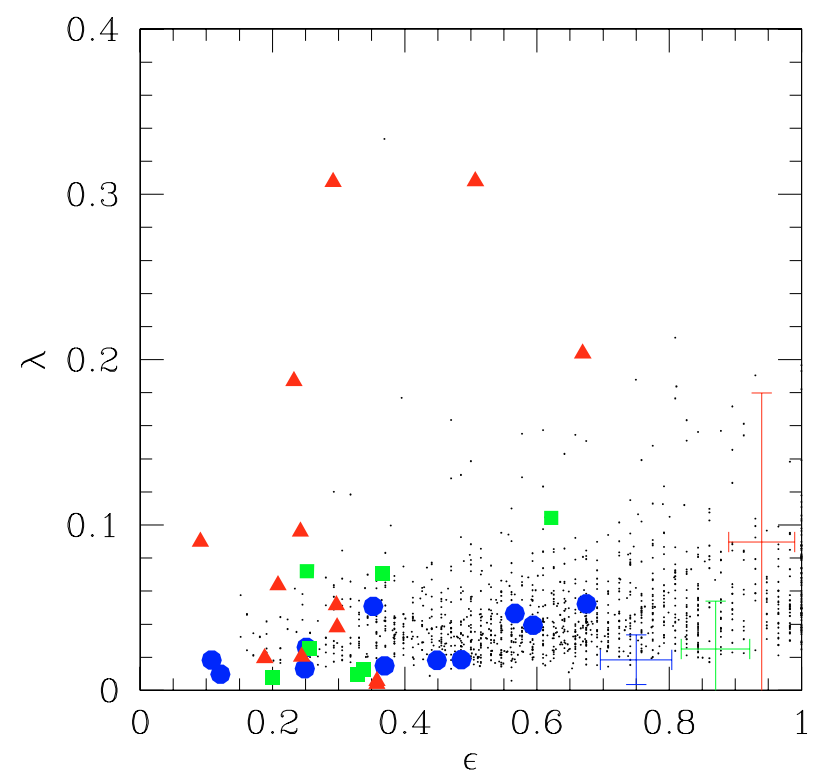

Fig. A.2. Spin parameter, $\lambda$, vs. ellipticity, $\epsilon$, in local and distant galaxy samples. Blue dots represent galaxies classified as rotating disks, green squares represent those classified as perturbed rotators, and red triangles represent galaxies with complex kinematics. Black dots represent the local sample of Courteau (1997) and Mathewson et al. (1992). The medians are indicated by the position of the median uncertainties, and distant galaxies are indicated on the right side of the plot (each being represented by matching colors). Errors include both standard propagation of error, and a bootstrap estimation of the error associated with the determination of the median itself.

of these two mechanisms. Both perturbed rotations and objects with complex kinematics show higher dispersion in the spin parameters than rotating disks (both local and distant). Part of this dispersion is probably due to the rotation velocity being underestimated for the galaxies that are not dynamically relaxed (see above). It could be tempting to claim this for an increasing dispersion among the three dynamical classes, from rotating disks 
to objects with complex kinematics, as was seen in the specific angular momentum, in agreement with a random-walk model driven by mergers. However, uncertainty also increases from rotating disks to kinematically complex galaxies, and it then becomes very difficult to firmly claim such an evolution in the dispersions. Although it seems possible to measure $\lambda$ for local galaxies, its derivation seems still very difficult for more distant objects.

\section{References}

Abadi, M. G., Navarro, J. F., Steinmetz, M., \& Eke, V. R. 2003, ApJ, 591, 499 Barnes, J., \& Efstathiou, G. 1987, ApJ, 319, 575

Bender, R., Saglia, R. P., \& Gerhard, O. E. 1994, MNRAS, 269, 785

Binney, J. 2005, MNRAS, 363, 937

Binney, J., \& Merrifield, M. 1989, Galactic Astronomy (Princeton, NJ, USA Princeton University Press)

Blecha, A., Cayatte, V., North, P., et al. 2000, Optical and IR Telescope Instrumentation and Detectors, ed. M. Iye, \& A. F. Moorwood, SPIE Proc., 4008, 467

Böhm, A., Ziegler, B. L., Saglia, R. P., et al. 2004, A\&A, 420, 97

Bottema, R. 1993, A\&A, 275, 16

Bullock, J. S., Dekel, A., Kolatt, T. S., et al. 2001, ApJ, 555, 240

Chen, D. N., Jing, Y. P., \& Yoshikaw, K. 2003, ApJ, 597, 35

Conselice, C. J., Bundy, K., Ellis, R. S., et al. 2005, ApJ, 628, 160

Corsini, E. M., Pizzella, A., Coccato, L., \& Bertola, F. 2003, A\&A, 408, 873

Courteau, S. 1997, AJ, 114, 2402

Cox, T. J., Primack, J., Jonsson, P., \& Somerville, R. S. 2004, ApJ, 607, L87

Cox, T. J., Jonsson, P., Primack, P., \& Somerville, R. S. 2006, MNRAS, 373, 1013

D’Onghia, E., Burkert, A., Murante, G., \& Khochfar, S. 2006, MNRAS, submitted [arXiv: astro-ph/0602005]

Davies, R. L., Efstathiou, G., Fall, S. M., Illingworth, G., \& Schechter, P. L. 1983, ApJ, 266, 41

Dekel, A., \& Birnboim, Y. 2006, MNRAS, in press [arXiv: astro-ph/0412300]

Drory, N., Salvato, M., Gabasch, A., et al. 2005, ApJ, 619, L131

Dutton, A. A., van den Bosch, F. C., Dekel, A., \& Courteau, S. 2006, ApJ, submitted [arXiv: astro-ph/0604553]

Eisenstein, D. J., \& Loeb, A. 1996, ApJ, 459, 432

Fall, S. M., \& Efstathiou, G. 1980, MNRAS, 193, 189

Flores, H., Hammer, F., Puech, M., Amram, P., \& Balkowski, C. 2006, A\&A, in press [arXiv: astro-ph/0603563], Paper I

Förster Schreiber, N. M., Genzel, R., Lehnert, M. D., et al. 2006, ApJ, in press [arXiv: astro-ph/0603559]

Gardner, J. P. 2001, ApJ, 557, 616

Gilmore, G., Wyse, R. F. G., \& Norris, J. E. 2002, ApJ, 574, L39

Governato, F., Willman, B., Mayer, L., et al. 2006, MNRAS, submitted [arXiv: astro-ph/0602351]

Hammer, F., Gruel, N., Thuan, T. X., Flores, H., \& Infante, L. 2001, ApJ, 550, 570

Hammer, F., Flores, H., Elbaz, D., et al. 2005, A\&A, 430, 115

Hammer, F., Lehnert, M. D., Puech, M., Flores, H., \& Liang, Y. C. 2006, Messenger, 123, 41

Heavens, A. F., \& Jimenez, R. 1999, MNRAS, 305, 770

Hernandez, X., \& Cervantes-Sodi, B. 2006, MNRAS, 368, 351

Hetznecker, H., \& Burkert, A. 2006, MNRAS, 370, 1905

Hoyle, F. 1951, in Problems of Cosmical Aerodynamics, Proceedings of the Symposium on the Motion of Gaseous Masses of Cosmical Dimensions held in Paris, France, August 1949, ed. J. M. Burgers, \& H. C. van de Hulst (Dayton: Central Air Documents Office), 195
Hunter, D. A., Rubin, V. C., Swaters, R. A., Sparke, L. S., \& Levine, S. E. 2005, ApJ, 634, 281

Kereš, D., Katz, N., Weinberg, D. H., \& Davé, R. 2005, MNRAS, 363, 2

Le Fèvre, O., Abraham, R., Lilly, S. J., et al. 2000, MNRAS, 311, 565

Lemson, G., \& Kauffmann, G. 1999, MNRAS, 302, 111

Lotz, J. M., Madau, P., Giavalisco, M., Primack, J., \& Ferguson, H. C. 2006, ApJ, 636, 592

Mathewson, D. S., Ford, V. L., \& Buchhorn, M. 1992, ApJS, 81, 413

Maller, A. H., \& Dekel, A. 2002, MNRAS, 335, 487

Maller, A. H., \& Bullock, J. S. 2004, MNRAS, 355, 694

Maller, A. H., Dekel, A., \& Somerville, R. 2002, MNRAS, 329, 423

McGaugh, S. S. 2005, ApJ, 632, 859

Mestel, L. 1963, MNRAS, 126, 553

Mo, H. J., Mao, S., \& White, S. D. M. 1998, MNRAS, 295, 31

Nesvadba, N. P. H., Lehnert, M. D., Eisenhauer, F., et al. 2006, ApJ, in press [arXiv: astro-ph/0606527]

Okamoto, T., Eke, V. R., Frenk, C. S., \& Jenkins, A. 2005, MNRAS, 363, 1299

Palunas, P., \& Williams, T. B. 2000, AJ, 120, 2884

Peebles, P. J. E. 1969, ApJ, 155, 393

Peirani, S., Mohayaee, R., \& de Freitas Pacheco, J. A. 2004, MNRAS, 348, 921

Pizzella, A., Corsini, E. M., Vega Beltrán, J. C., \& Bertola, F. 2004, A\&A, 424, 447

Porciani, C., Dekel, A., \& Hoffman, Y. 2002, MNRAS, 332, 325

Portinari, L., \& Sommer-Larson, J., MNRAS, submitted [arXiv: astro-ph/0606531]

Press, W. H., Flannery, B. P., \& Teukolsky, S. A., et al. 1989, Numerical recipes in C (Cambridge, UK: Cambridge University Press)

Puech, M., Hammer, F., Flores, H., Östlin, G., \& Marquart, T. 2006a, A\&A, in press ([arXiv: astro-ph/0603562], Paper II)

Puech, M., Flores, H., Hammer, F., \& Lehnert M. 2006b, A\&A, in press [arXiv:astro-ph/0603564]

Ravikumar, C., Flores, H., Proust, D., Puech, M., et al. 2006, A\&A, submitted

Robertson, B., Hernquist, L., Bullock, J. S., et al. 2005, ApJ, in press [arXiv: astro-ph/0503369]

Schade, D., Lilly, S. J., Le Fevre, O., Hammer, F., \& Crampton, D. 1996, ApJ, 464, 79

Syer, D., Mao, S., \& Mo, H. J. 1999, MNRAS, 305, 357

Silk, J. 1997, ApJ, 481, 703

Simard, L., \& Pritchet, C. J. 1998, ApJ, 505, 96

Simard, L., Willmer, C. N. A., Vogt, N. P., et al. 2002, ApJS, 142, 1

Springel, V., \& Hernquist, L. 2005, ApJ, 622, L9

Steinmetz, M., \& Navarro, J. 1999, ApJ, 513, 555

Strömberg, G. 1934, ApJ, 79, 460

Tonini, C., Lapi, A., Shankar, F., \& Salucci, P. 2006, ApJ, 638, L13

van den Bosch, F. C. 2000, ApJ, 530, 177

van den Bosch, F. C. 2001, MNRAS, 327, 1334

van den Bosch, F. C. 2002, MNRAS, 332, 456

van den Bosch, F. C., Abel, T., Croft, R. A. C., Hernquist, L., \& White, S. D. M. 2002, ApJ, 576, 21

van den Bosch, F. C., Abel, T., \& Hernquist, L. 2003, MNRAS, 346, 177

Vega Beltrán, J. C., Pizzella, A., Corsini, E. M., et al. 2001, A\&A, 374, 394

Velazquez, H., \& White, S. D. M. 1999, MNRAS, 304, 254

Vitvitska, M., Klypin, A. A., Kravtsov, A. V., et al. 2002, ApJ, 581, 799

Walker, I. R., Mihos, J. C., \& Hernquist, L. 1996, ApJ, 460, 121

Wechsler, R. H., Bullock, J. S., Primack, J. R., Kravtsov, A. V., \& Dekel, A. 2002, ApJ, 568, 52

White, S. D. M. 1984, ApJ, 286, 38

White, S. D. M., \& Rees, M. J. 1978, MNRAS, 183, 341

Wyse, R. F. G., Gilmore, G., Norris, J. E., et al. 2006, ApJ, 639, L13

Yoachim, P., \& Dalcanton, J. J. 2005, ApJ, 624, 701

Yoachim, P., \& Dalcanton, J. J. 2006, AJ, 131, 226

Ziegler, B. L., Böhm, A., Fricke, K. J., et al. 2002, ApJ, 564, L69 